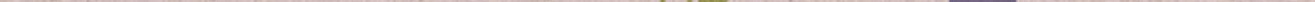




\title{
Participación y empoderamiento de las mujeres en las cooperativas: un estudio sobre la participación de mujeres en cooperativas de la agricultura familiar en Brasil
}

\author{
Women Participation and Empowerment \\ in Cooperative Organizations in Brazil: \\ An in-depth study
}

Silvia A. Zimmermann* | Marcos de Oliveira Garcias** | Dirceu Basso***

\begin{abstract}
Resumen
La práctica cooperativa es ancestral y el movimiento cooperativo ha avanzado en busca de respuestas a los desafíos y formas de pensar sobre el trabajo cooperativo. Entre las cuestiones actuales del cooperativismo destacamos la comprensión de la participación de las mujeres en el manejo de las iniciativas cooperativas, sus limitaciones y motivaciones en un intento por comprender qué proyectos o políticas pueden contribuir a una mayor participación de las mujeres en estos espacios. Por demanda de la comisión de género de la Reunión Especializada sobre Agricultura Familiar del Mercosur (REAF-Mercosur) se llevó a cabo una investigación con el objetivo de verificar con más detalle cuestiones relacionadas la participación de las mujeres en las cooperativas. Se entiende que las mujeres participan en la cooperativa, pero que la percepción de esta participación ocurre, o se refuerza, en el momento de la participan más activa en la producción del producto o servicio que son intercambiados con la cooperativa.
\end{abstract}

Palabras clave: cooperativismo; empoderamiento femenino; Agricultura familiar.

\begin{abstract}
Cooperative practice is ancestral and the cooperative movement has advanced in search of answers to the challenges and ways of thinking about cooperativism. Among the current issues of cooperativism, one of them is the understanding of women's participation in the management of cooperative initiatives, their limitations and motivations in an attempt to understand what projects or policies can contribute to a greater participation of women in these spaces. At the request of the Gender Commission of the Specialized Meeting on Family Farming of Mercosur (REAF-Mercosur) an investigation was carried out in order to verify issues related to the participation of women in cooperatives. It is understood that women participate in the cooperative, but that the perception of this participation occurs, or is reinforced, when they participate more actively in the production of the product or service, that they are negotiate with the cooperative.
\end{abstract}

Keywords: cooperativism; female empowerment; family farming.

\footnotetext{
* Profesora de la Universidad Federal de la Integración Latino-Americana (UNILA). Doctorado en Sociología, Desarrollo, Agricultura y Sociedad por la Universidad Federal Rural de Rio de Janeiro (CPDA/UFRRJ). https://orcid.org/0000-0003-2318-2743. E-mail: silvia.zimmermann@unila.edu.br

** Profesor de la Universidad Federal de la Integración Latino-Americana (UNILA). Doctorado en Economía Aplicada por la Escuela Superior de Agricultura Luiz de Queiroz de la Universidad de São Paulo (ESALQ-USP). https://orcid.org/0000-0002-4178-6842.E-mail:marcos.garcias@unila.edu.br

*** Profesor de la Universidad Federal de la Integración Latino-Americana (UNILA). Doctorado en Desarrollo Rural por la Universidad Federal de Rio Grande do Sul (PGDR/UFRGS). https://orcid.org/0000-0003-1487-6049.

E-mail:dirceu.basso@unila.edu.br
}

http://dx.doi.org/10.51861/ded.dmv.1.011 


\section{INTRODUCCIÓN}

El cooperativismo se entiende como una forma ancestral de hacer las cosas en la sociabilidad humana y la expansión del movimiento cooperativo es una respuesta a los desafíos y formas de pensar sobre el trabajo cooperativo (MAYO, 2017), siendo la perspectiva cooperativa un objeto de interés para las iniciativas públicas o privadas. Existen actualmente innumerables experiencias en todo el mundo, en amplios sectores.

Un tema actual en el estudio del cooperativismo es la comprensión de la participación de las mujeres en el manejo de las iniciativas cooperativas (DUGUID \& WEBER, 2016; OIT, 2016), donde de modo general, la literatura cooperativa de mujeres aborda los siguientes temas: i) acceso al mercado laboral y oportunidades laborales creadas con otras mujeres, ii) combate de las normas sociales, culturales y religiosas opresivas para las mujeres, iii) ampliación del papel de la mujer más allá de los roles domésticos y reproductivos, iv) mejora de las condiciones laborales, incluyendo trabajo decente, igualdad salarial y protección social, v) desarrollo social, como capacitación, liderazgo y desarrollo de la autoestima, vi) falta de educación formal y capacitación para que las mujeres tengan éxito en los negocios, vii) transición del trabajo en la economía informal a la economía formal, viii) ciertos sectores de trabajo, como el trabajo doméstico y los servicios de asistencia, en particular, ix) oportunidades que vienen con la capacidad de una mujer para ser independiente $\mathrm{y}, \mathrm{x}$ ) la naturaleza democrática de las cooperativas permite a las mujeres practicar la participación y participar en la toma de decisiones (DUGUID \& WEBER, 2016: 22).

En el cooperativismo para agricultura familiar el tema presente son las limitaciones que enfrentan estas mujeres y las estrategias que pueden contribuir a su rol cooperativo para el desarrollo rural (ZIMMERMANN, GARCIAS \& BASSO, 2019; SAGGIN \& ALVES, 2019; GRADE \& BASSO, 2019; SOUZA \& DE MARCH, 2018; VOLLES \& BONA, 2016; BUENO \& KNUPPEL, 2016; CARRETERO \& AVELLO, 2011). Los estudios, basados en entrevistas estructuradas o semiestructuradas, cuestionarios y análisis cualitativos y cuantitativos, desde diferentes referencias teóricas, reconocen el cooperativismo como una institución para superar la desigualdad de género, también en áreas rurales; analizan la inserción de mujeres en los consejos de las cooperativas de crédito; analizan cómo se produce el empoderamiento de las mujeres en las cooperativas; y tratan de identificar el perfil de las mujeres que participan en el movimiento cooperativo.

En general, los trabajos mencionados afirman que la participación de las mujeres en las cooperativas genera empoderamiento, en la perspectiva de promover las libertades. En este sentido, el empoderamiento de las mujeres y 
sus organizaciones les permite tomar el control de sus vidas y destinos, de los asuntos en los que están involucradas, de las reflexiones y conciencia, para la generación de su autonomía frente al mundo y para su autodeterminación (JALIL, 2013). La mayoría de los estudios mencionados están destinados a colaborar en el diseño de políticas públicas que puedan contribuir a una mayor participación de las mujeres en las cooperativas.

En este sentido, a partir de una demanda de la comisión de género de la Reunión Especializada sobre Agricultura Familiar del Mercosur (REAFMercosur) realizamos una encuesta con el objetivo de verificar con mayor profundidad la participación de las mujeres en las cooperativas, con el fin de subsidiar debates en la Comisión Técnica de Género del REAF Mercosur. Al mismo tiempo, se buscó un enfoque original en Brasil en términos de la muestra de investigación y de la información recopilada, en vista de un cuestionario semiestructurado basado en una encuesta similar realizada por la Confederación Uruguaya de Entidades Cooperativas (Cudecoop) en 109 mujeres, en octubre de 2017, en Uruguay (CUDECOOP, 2017). La investigación tuvo como propósito garantizar dados profundados, para atender a demanda por más investigaciones sobre la participación de las mujeres en experiencias cooperativas, carentes de dados, métricas y dados desagregados por sexo (DUGUID \& WEBER, 2016; OIT, 2016).

La REAF Mercosur fue creada en 2004, siendo una de las quince reuniones especializadas en el Mercosur. Reúne productores familiares, organizaciones e instituciones rurales de la región, con el objetivo de generar un marco de políticas públicas regionales para la agricultura familiar y promover la facilitación del comercio de la producción de base familiar entre los países miembros (REAF, 2016). Dentro de la REAF hay grupos de trabajo sobre temas específicos, a saber, acceso a la tierra y reforma agraria; juventud rural; género, gestión de riesgos y cambios climáticos; y facilitación del comercio.

La REAF propone la idea de transversalidad de género, como principio para la integración de las políticas públicas, siendo esta cuestión objeto de una serie de directrices aprobadas por el Comercio del Mercado Común (CMC), que recomienda a los países del bloque la adopción de dicha noción en el conjunto de las políticas para la agricultura familiar, como también la promoción de acciones positivas específicas de mujeres y el estímulo a la participación social para la implementación de políticas que garanticen la igualdad de género. A partir de la aprobación de las directrices sobre igualdad de género y de la creación del Programa Regional de Fortalecimiento Institucional de las Políticas de Igualdad de Género en la Agricultura Familiar, el GT de Género inició una acción coordinada y sistemática de diálogo entre los gobiernos y la sociedad civil para incorporar de manera efectiva un 
enfoque de género en las políticas públicas de los Estados miembros (REAF, 2016). Entre las estrategias para las políticas públicas para la región, la REAF ha incluido en la agenda sugerencia de acciones de estímulo al asociativismo y al cooperativismo, siendo estas acciones también sensibles a transversalidad de género.

La investigación se llevó a cabo dentro del alcance del 5o Encuentro de Jóvenes Cooperativos, 1 Encuentro de Mujeres Rurales del Mercosur y el 1o Seminario Internacional sobre Género y Generación de Cooperativismo Solidario, organizado por la Unión de Cooperativas de Agricultura y Economía Familiar y Solidaria (Unicafes), en asociación con la REAF Mercosur, el Observatorio Latinoamericano de Agricultura Familiar e Itaipu Binacional, en un evento celebrado entre el 17 y el 19 de octubre de 2018, en Medianeira, Paraná, Brasil. El 19 de octubre, el día en que se realizó la encuesta, el evento involucró principalmente a la audiencia femenina para abordar el tema del cooperativismo y el tema de género. Ese día, aproximadamente 1100 mujeres de entidades vinculadas a la Agricultura Familiar y la Economía Solidaria de Paraná y otros estados de Brasil, y países del Mercosur más amplio (Paraguay, Argentina, Uruguay, Chile, Colombia y Ecuador) participaron del evento, además de representantes de Perú. Destacamos que en el evento había una gran mayoría de mujeres de la región sur de Brasil, especialmente del Estado de Paraná.

Para comprender mejor el contexto destacamos que la Unicafes representa a las cooperativas de la agricultura familiar y de la economía solidaria a nivel nacional en Brasil. Nació en junio de 2005 y se propone la inclusión social, articulando iniciativas económicas que amplíen las oportunidades de trabajo, distribución de ingresos, producción de alimentos, mejoras en la calidad de vida, preservación de la biodiversidad y reducción de las desigualdades (UNICAFES, 2020). Hay 700 cooperativas de varias áreas (crédito, producción, transporte, trabajo, comercialización e infraestructura para la agricultura familiar y la economía solidaria y otras) asociadas a Unicafes distribuidas en las cinco regiones del país, siendo organizadas en 19 Unicafes regionales, que brindan asistencia y articulación cooperativas a los asociados (UNICAFES, 2020).

La investigación presenta un enfoque cualitativo y cuantitativo, con procedimientos de investigación de campo a través de entrevistas utilizando el método de encuesta. De esta manera, este trabajo es de naturaleza aplicada, con un objetivo exploratorio y explicativo, según la clasificación descrita por Triviños (1987). Se aplicó un cuestionario para 173 mujeres (15,7\% de los participantes), que respondieron 22 preguntas, abiertas y cerradas, sobre la participación de las mujeres en espacios cooperativos. Los resultados 
obtenidos con esta muestra son estadísticamente representativos a 90\% de confianza, con un intervalo de $6 \%$ para más o para menos. Con el fin de obtener análisis comparativos entre los países que participan en REAF, la mayoría de las preguntas fueron las mismas que en el cuestionario aplicado por Cudecoop (2017). La aplicación en el evento brasileño estuvo a cargo de estudiantes y profesores de la Universidad de la Integración Latino Americana (Unila), y los datos fueron digitalizados y trabajados en Excel $^{2}$.

Los resultados de la investigación son organizados en cinco secciones. La primera presenta el perfil de las mujeres entrevistadas, la edad y la ascendencia étnico-racial y el perfil de las cooperativas a las que están vinculadas, los productos y servicios, el tiempo dedicado y las actividades realizadas. La segunda presenta las percepciones de las mujeres sobre la participación, las dificultades y los factores que afectan la participación, especialmente la percepción sobre la relación entre las tareas domésticas y la participación en la cooperativa. La tercera presenta percepciones sobre la promoción de la participación en las cooperativas y sugerencias de las mujeres para revertir la baja participación encontrada trayendo reflexiones sobre acciones y políticas públicas. Por último, las consideraciones finales y las referencias bibliográficas ${ }^{3}$.

\section{EL PERFIL DE LAS MUJERES INVOLUCRADAS EN LA INVESTIGACIÓN, SUS COOPERATIVAS Y SUS PARTICIPACIONES}

La mayoría de las entrevistados (78\%) tienen más de 40 años. Una parte importante tiene más de 55 años (33\%), y dada la legislación laboral vigente en 2018, posiblemente estén jubiladas. Estos datos corroboran los estudios que abordan el fenómeno del envejecimiento de las mujeres que viven en zonas rurales, ya que casi la mitad de los participantes tienen más de 50 años.

Tabla 1 - Grupo de edad, 2018

\begin{tabular}{l|c|c}
\hline Edad & Quant. & \multicolumn{1}{c}{$\%$} \\
\hline 12-19 Años & 02 & 1,156 \\
\hline 20-29 Años & 10 & 5,78 \\
\hline 30-39 Años & 26 & 15,03 \\
\hline 40-49 Años & 47 & 27,17 \\
\hline 50-54 Años & 31 & 17,92 \\
\hline Más de 55 Años & 57 & 32,95 \\
\hline Total & 173 & 100 \\
\hline
\end{tabular}

Fuente: datos de la Investigación, 2018. 
Cuando se les preguntó sobre el género que identifican, el $82 \%$ indicó ser femenina y el $18 \%$ mujer. Surgieron algunas variaciones, como "mujer joven”, “mujer agricultora", “mujer heterosexual” y género "opuesto”. Es de destacar que la pregunta generó cierta sorpresa entre las mujeres y para algunas, incluso, molestias. El debate sobre el significado del género y sus implicaciones históricas (Scott, 1995) parece no ser parte de la vida cotidiana de las entrevistadas. Al mismo tiempo, la mayoría de las mujeres entrevistadas creía que tenían una etnia racial, el 67\% dijo que sí y el 33\% dijo que no. De las que respondieron que sí, hay una variedad de posibilidades: africana, alemana, asiática, blanca, campesina, gitana, española, europea, indígena, italiana, morena, polaca, portuguesa y rumana. También se mencionaron variaciones como alemán-italiano, alemán-indio, italiano-polaco, entre otras. Agrupadas estas situaciones se puede afirmar que hay un colectivo que se reconoce a sí mismo con ascendencia predominantemente europea (57\%), seguido de indígenas (23\%), afrobrasileños (16\%), asiáticos (2,5\%) y gitanos (1,5\%). Esta pregunta también mostró que no hay claridad entre las participantes sobre el significado de ascendencia étnica y las variaciones existentes.

La mayoría de las entrevistadas dijeron que estaban vinculadas a las cooperativas de crédito (56\%), seguida de las cooperativas agrícolas (32\%), las cooperativas sociales (5\%), de trabajo (5\%) y de consumo (1\%). En el rubro otros $(1 \%)$ se encuentran cooperativas de recicladores y consumidores. Es de destacar que las sucursales cooperativas de la Unicafes son diversas, con una mayor consolidación de la sucursal de crédito solidario en las últimas décadas.

Cuando se les preguntó sobre la actividad principal llevada a cabo por la Cooperativa participante, 139 mujeres indicaron el tema del crédito (60\%) (dicho por las mujeres como financiamiento, servicios financieros, CresolCooperativa de Crédito Solidario ${ }^{4}$, entre otras expresiones), lácteos (10\%), cereales $(9 \%)$, frutas $(6 \%)$, diversos $(6 \%)$, verduras $(4 \%)$, derivados de caña (2\%), servicios (2\%); juntos en un $1 \%$ hay aves, plantas medicinales, productos orgánicos y pan. Preguntadas sobre la principal actividad económica que ellas realizan junto la cooperativa, la mayoría comentó sobre el acceso a los servicios de crédito y bancarios (32\%). Una porción significativa vende leche $(26 \%)$ y cereales $(22 \%)$ en cooperativas, pero también soja, maíz, trigo, frijoles y sésamo. Entre las frutas (6\%), se mencionaron uvas y maracuyá. Casi la mitad de las entrevistadas se inserta en sistemas productivos relacionados con la actividad láctea y de cereales, pero es posible observar la existencia de otras actividades productivas, con énfasis en la producción de hortalizas y mandioca (6\%). En general, existe una importante diversidad productiva en los establecimientos familiares de las mujeres entrevistadas, 
siendo extremadamente importante la participación de las mujeres en el trabajo. Cinco mujeres (4\%) indicaron que el producto que entregan a la cooperativa es comercializado a través de alimentación escolar.

La mayoría de las mujeres participan de 1 a 5 horas (72\%) en los espacios de gestión de la cooperativa; 5-10 horas (22\%); más de 10 horas (6\%). El tiempo dedicado a la cooperativa tiene limitaciones de diferentes órdenes, según las respuestas de las mujeres:

Cuadro 01- Respuestas de las mujeres a los factores que afectan la participación, 2018

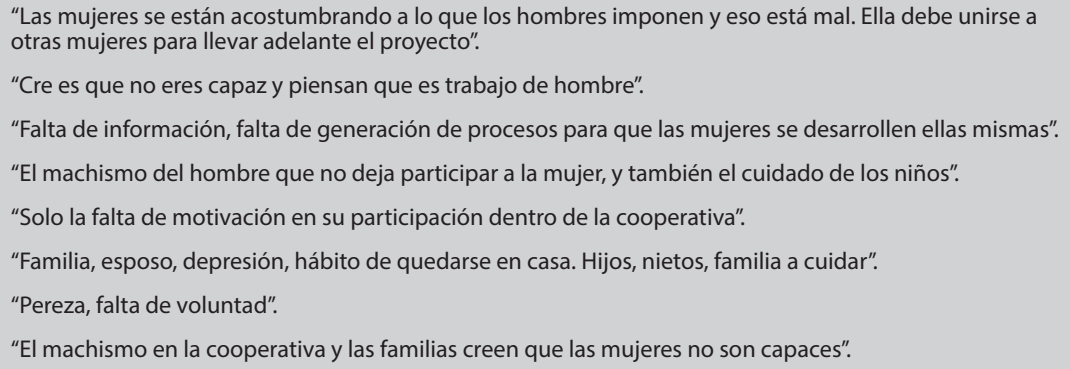

Fuente: datos de la Investigación, 2018

$\mathrm{Al}$ analizar el papel de las cooperativas en el despertar de la participación y la acción política de las mujeres en las zonas rurales, Grade y Basso (2019) presenta que el cooperativismo contribuye a construir lazos de pertenencia y solidaridad, que permiten superar los procesos de exclusión y mejoría de las condiciones de vida de las mujeres en el campo.

$\mathrm{Al}$ ser indagadas sobre la participación, apenas 61 mujeres (35\%) mencionaron participar en alguna forma de Comisiones dentro de la Cooperativa. De estas mujeres, el 41\% tiene más de 55 años y el 26\% tiene entre 40 y 49 años. Prácticamente no había mujeres jóvenes en las comisiones. De hecho, en oportunidad de la aplicación del cuestionario hubo baja participación de personas jóvenes, identificándose solo 4 mujeres (6\%) menores de 29 años en ese momento. Entre las comisiones, proyectos y acciones de cooperación en las que participan, los entrevistadas mencionan: Tercera Edad, Información, Ayuda general, Club de madres, Gestión, Producción, Comercio y Gestión, Administrativo, Género y Generación, De productores de leche, Permanente, Deliberativo, Impuestos, Cuotas, Jornada de campo, Dirección, Finanzas, Financiación, Gestión, Grupo de Mujeres, Jóvenes, Mujeres, Liderazgo de mujeres / mujeres trabajadoras y Consejo de la feria. Encontramos una gran variación entre las cooperativas sobre las formas de gestión de las actividades. 
Hay 34 mujeres (20\%) que dijeron que participan en una comisión directiva en la cooperativa, en puestos como Agente, Consejero Fiscal, Coordinación, Directora Consejera, Gerente General, Presidencia, Presidente, Secretaria, Consejero, Tesorera. Doce mujeres (37.5\%) son miembros de consejos o coordinaciones; 8 (25\%) declaran trabajo en la Presidencia y vicepresidencia; 2 mujeres (6\%) trabajan en Secretarias y 3 (9\%) son tesoreras.

Para Duguid y Weber (2016, p. 18) las cooperativas generalmente reflejan las mismas relaciones de poder que la sociedad en la que viven y esta réplica de las relaciones de poder también ocurre en las cooperativas Sin embargo, existe cierta evidencia de que pueden producirse resultados positivos de empoderamiento para las mujeres, sobretodo con las mujeres en puestos de liderazgo en campos tradicionalmente dominados por hombres (por ejemplo, finanzas).

La mayoría de las mujeres que participan en el comité directivo de las cooperativas y que respondieron la encuestas tienen más de 40 años, son $85 \%$ (29 mujeres), y las mayores de 55 representan el 47\% (16 mujeres). Por el contrario, las mujeres más jóvenes, o menores de 40 años, son las que menos participan en puestos directivos y representan el 15\% (5 mujeres). Estos datos reafirman el protagonismo de las mujeres mayores de 40 años en las actividades de la cooperativa, una situación ya evidenciada en el estudio de Volles y Bonn (2016). Al entrevistar a 15 mujeres en la actividad de dirección de Cresol (Cooperativa de Crédito solidário), estos autores identificaron que el 66\% de las entrevistadas tenía entre 41 y 60 años. Saggin y Alves (2019), en un estudio sobre el perfil de las mujeres que participaron en el Congreso de los 10 años de creación de la Unicafes, celebrada en 2015, también encontraron que el grupo de edad más activo de las mujeres que participan en los movimientos de mujeres, con énfasis en el movimiento cooperativo, está entre 41 y 65 años. Por último, se destaca que en el comité directivo el mayor tiempo dedicado a la cooperativa es entre 1-5 horas (21 mujeres o 62\%); 5 - 10 horas (10 mujeres o $29 \%$ ) y más de 10 horas (3 mujeres o $9 \%$ ).

\section{DIFICULTADES Y POSIBILIDADES DE PARTICIPACIÓN DE LAS MUJERES EN COOPERATIVAS}

La participación en los espacios de dirección es reconocida como la más difícil de ser ocupada por mujeres en cooperativas, mientras que las funciones de comisiones son ocupadas más frecuentemente por mujeres. Según Volles y Bona (2016: 18), "a pesar de los avances, hay una escasa participación latente de las mujeres en los consejos o dirección de las cooperativas, especialmente en los puestos más altos”. También, en la percepción de las entrevistadas, 
es más difícil para las mujeres asumir puestos gerenciales (56\%), espacios de trabajo (49\%) y comisiones (38\%).

Esta situación ocurre debido a las innumerables dificultades mencionadas por las mujeres, con énfasis en el tiempo dedicado a las tareas domésticas y la falta de capacitación o formación. El estudio de la Cudecoop (2017) presenta que $15 \%$ de las encuestadas señaló que las dificultades para acceder a los puestos de dirección están dadas por una desvalorización por género internamente en las cooperativas.

En la investigación también se menciona la falta de motivación, inseguridad y autoafirmación. Notoriamente, las mujeres no abandonan las tareas domésticas pero agregan más trabajo cuando se unen a grupos o asociaciones productivas y estas inserciones requieren el establecimiento de relaciones políticas e institucionales, saber cómo lidiar con el crédito y el endeudamiento, comercializar la producción, "aprender a hablar" y saber "lidiar con el dinero". Desafíos que intimidan a muchas mujeres (BRUNO ET AL, 2013:227).

Tabla 02 - Percepción sobre los factores que dificultan la participación, 2018

\begin{tabular}{l|c|c|c|c|c|c}
\hline \multirow{2}{*}{ Opções } & \multicolumn{2}{|c|}{ Afecta Mucho } & \multicolumn{2}{l|}{ Afecta Poco } & \multicolumn{2}{c}{ No Afecta } \\
\cline { 2 - 8 } & Quant. & $\%$ & Quant. & $\%$ & Quant. & $\%$ \\
\hline Tareas y cuidados Domésticos & 72 & 63 & 30 & 26 & 13 & 11 \\
\hline Inseguridad y Autoafirmación & 37 & 47 & 26 & 33 & 16 & 20 \\
\hline Falta de Formación & 64 & 63 & 27 & 26 & 11 & 11 \\
\hline Falta de Motivación & 51 & 55 & 28 & 30 & 13 & 14 \\
\hline
\end{tabular}

Fuente: datos de la Investigación, 2018.

Una parte importante de las mujeres no se siente devaluada al presentar su opinión en la cooperativa (33\%). Sin embargo, una parte importante expresa que sienten que su opinión no se tiene en cuenta (39\%), se sienten cuestionadas por no tener capacitación (41\%) y no se sienten cómoda en los espacios de conducción (43\%). El problema de falta de formación aparece en un 5\% de las respuestas como una limitación para la participación personal en la investigación del Cudecoop (2017), por "no entender de cooperativismo"; "me es difícil entender sobre finanzas” y "creer no estar capacitada”. Los datos de la investigación de Volles y Bona (2016) indican que los consejeros respetan la opinión de las mujeres, sin embargo, todavía hay evidencia de ofensa y crítica entre las entrevistadas de esta investigación (22\%).

Así, entre los factores que las entrevistadas creen que afectan la participación de las mujeres se destacan la falta de apoyo, oportunidades, capacitación 
e información (35\%); machismo (22\%); inseguridad, autoestima y auto valorización (12\%); falta de conciencia, comodidad, voluntad o interés (11\%); cuidado de niños y familias (8\%); distancia para viajar a la cooperativa, dependencia para moverse y no saber conducir (8\%); falta de tiempo (5\%); tareas domésticas y cuidados en el hogar (4\%); problemas de salud (4\%); influencia de la religión (2\%). La acumulación de actividades laborales en las cooperativas, las funciones maternas y las funciones domésticas, a menudo se mencionan repetidamente en investigaciones sobre la participación de las mujeres en las cooperativas (SOUZA \& de MARCH, 2018; VOLLES \& BONA, 2016; BUENO \& KNUPPEL, 2016).

En la investigación del Cudecoop (2017:08), entre las dificultades de participación, el 19\% de las mujeres indicaron que su dificultad de participación es producto de tradiciones dadas en el orden interno de las cooperativas, y que los espacios que están destinados a cada asociado según sea mujer o varón, siendo que, culturalmente, se permite que los varones integren los órganos de decisión y las mujeres los espacios de fomento. En nuestra investigación, el cuestionario aplicado tenía una pregunta específica sobre las tareas domésticas que obtuvo una respuesta equilibrada: 88 mujeres $(51 \%)$ creen que las tareas domésticas limitan sus posibilidades de participación, mientras que 84 (49\%) creen que no. Cuando se les preguntó sobre la cantidad de horas diarias dedicadas a realizar tareas y cuidados domésticos no remunerados, la mayoría indicó $1-5$ horas (39\%); seguido de dedicación de 10 a 15 horas (33\%); 5 - 10 horas (14\%); más de 15 horas (13\%). Es posible que parte del tiempo dedicado a tareas domésticas incluya actividades agrícolas.

\section{PROMOCIÓN DE LA PARTICIPACIÓN EN LA COOPERATIVA - REFLEXIONES SOBRE ACCIONES Y POLİTICAS PÚBLICAS}

En una investigación coordenada por Schincariol McMurtry y McMurtry (2015) con un survey on-line a expertos y especialistas de movimientos cooperativos, laborales y de mujeres de distintas regiones del mundo, la mayoría (más del 75\%) de las personas encuestadas afirmaron que las cooperativas han mejorado la participación femenina en los últimos 20 años (DUGUID \& WEBER, 2016). Estos datos son utilizados por la Organización Internacional del Trabajo (OIT) y la Alianza Cooperativa Internacional (ACI), para pensar estrategias en la consolidación de la participación femenina en las cooperativas.

En nuestra investigación, la mayoría de las mujeres (59\%) indican que el tema de género se ha hecho factible, discutido o abordado en su cooperativa. Afirman que el tema de género se ha tratado directa o indirectamente en 
debates en la cooperativa a través de diversas actividades y eventos: cursos, conferencias, consejos y asambleas, reuniones, grupos de mujeres, organización de equipos, mesas redondas, conferencias, programas para mujeres, reuniones, seminarios, actividades de planificación y desarrollo de proyectos con mujeres, actividades para organizar cooperativas de mujeres, capacitación y actividades para valorar a las mujeres. Las mujeres también destacan la necesidad de actividades motivadoras, que valoren la autoestima y la participación de las mujeres, que promuevan la igualdad de derechos para las mujeres en las cooperativas, eventos que involucren la participación familiar, eventos mixtos, que involucren a hombres y mujeres jóvenes para debatir cuestiones de género y paridad en el trabajo. También actividades informativas, promoción de alianzas interinstitucionales y reuniones participativas, reuniones que involucren el tema de la salud de las mujeres y la alimentación.

Por otro lado, una mujer comenta que, aunque existe un debate de género en la cooperativa, "esto no se lleva a cabo en la práctica", lo que indica la necesidad de incorporar mecanismos que promuevan las prácticas de participación de las mujeres en las cooperativas. Por ejemplo, se sugiere la necesidad de tener cambios en los estatutos de las cooperativas, con artículos que pueden definir un mínimo de participación y de mujeres en la dirección. Al mismo tiempo, las mujeres comentaron que aunque hay acciones de género, "podría haber más”, ya que las actividades se llevan a cabo "solo en eventos externos" a la cooperativa. Dos mujeres mencionan no tener tiempo para capacitarse y que las cooperativas "tienen espacios de trabajo, pero no ayudan".

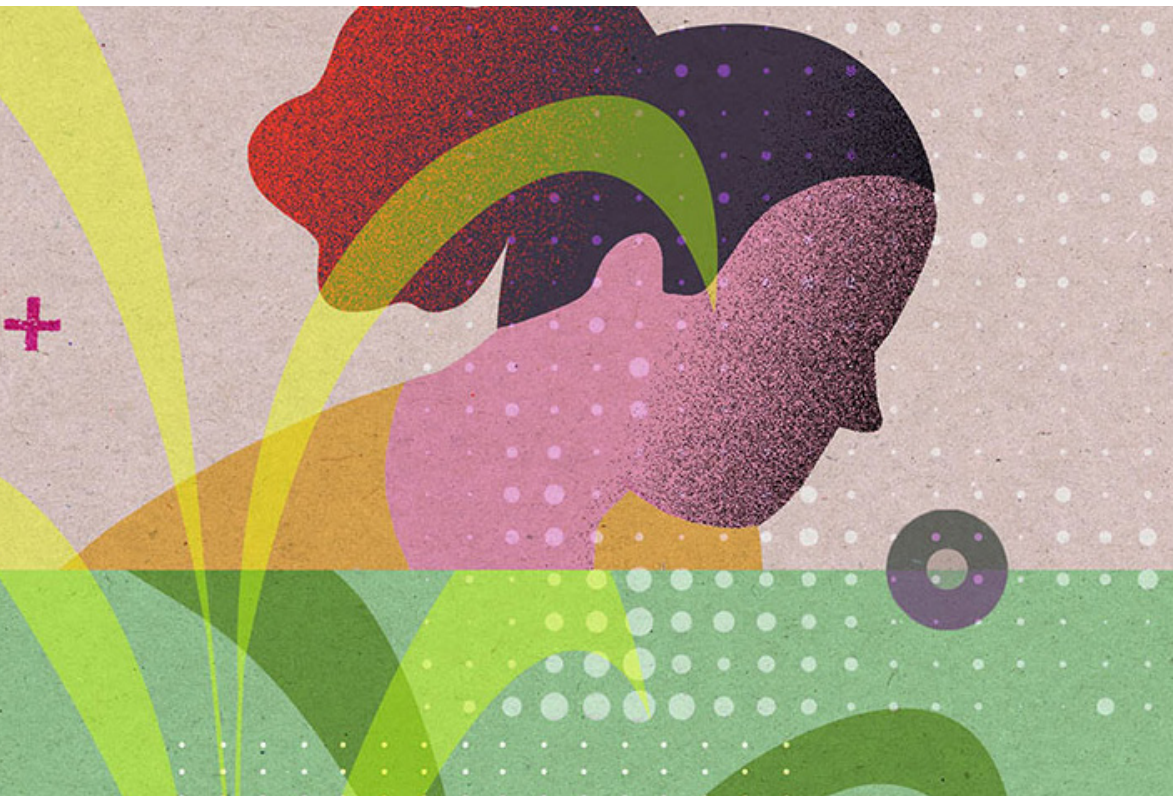


La mayoría de las mujeres (57\%) indica que han sido invitadas a revertir parcial o totalmente las dificultades en la participación de las mujeres en su cooperativa, mientras que el $30 \%$ dice que no y el 13\% indica que su cooperativa no tiene dificultades. Aquellas que dicen no ser invitadas son en su mayoría mayores de 40 años (38 mujeres, 75\%).

Entre los factores que afectan la participación de las mujeres, se menciona la necesidad de acciones para alentar la participación; motivación para generar iniciativa y empoderar a las mujeres; capacitación y desarrollo de capacidades; creación y espacios de inclusión y diálogo con mujeres en la cooperativa; recursos financieros para acciones de mujeres; guarderías para garantizar el cuidado de los niños; transporte para participar de las actividades de la cooperativa; división de las tareas del hogar para que tengan más tiempo para participar en la cooperativa; acciones en las cooperativas que motiven la participación de las mujeres; políticas públicas dirigidas a las mujeres; mayor difusión de las actividades de la cooperativa entre las mujeres y, por último, horarios de actividades más accesibles a los horarios de disponibilidad de las mujeres.

Creemos que las acciones e iniciativas de las cooperativas impactan sobre el empoderamiento femenino, como muestra el estudio de Vedana (2020) hecho en una cooperativa agroindustrial en la región oeste del Paraná, Brasil. La investigación aplicó un cuestionario adaptado del Women's Empowerment in Agriculture Index (WEAI), para 150 parejas (300 personas), divididas en dos grupos, uno para mujeres que participan en un Programa de la cooperativa para liderazgo femenino y un grupo que no participa. Los resultados indicaron que las mujeres encuestadas están empoderadas, ya que cumplen con la adecuación en más del $80 \%$ de los indicadores ponderados; sin embargo, la puntuación de las mujeres en el grupo que participa del Programa es más alta que la del grupo que no participa. Los resultados sugieren que la iniciativa de formación y capacitación de la cooperativa para las mujeres contribuye a la creación de condiciones favorables para una mayor participación de las mujeres en el proceso de toma de decisiones relacionadas con la agricultura.

En grande medida nuestra reflexión acerca de los resultados de la investigación hecha sobre la participación de las mujeres en las cooperativas cree ser necesarias la creación y promoción de acciones y políticas públicas gubernamentales. Así, los resultados de nuestra investigación coinciden con la investigación de la OIT (2016:21), donde la mayoría (88\%) de los entrevistados afirmó que el Estado tiene un papel importante para lograr la igualdad de género y el empoderamiento de la mujer en toda la sociedad y en la formulación de políticas que apoyen a las cooperativas. $85 \%$ de los entre- 
vistados considera que "ayudar a las mujeres emprendedoras" debe ser una de las prioridades de los gobiernos. En la misma investigación de la OIT las encuestadas recomendaran que los gobiernos adopten prácticas equitativas que propicien el desarrollo cooperativo y pueda legitimar el modelo cooperativo, enfatizando las siguientes prácticas: i) revisar, enmendar o idear leyes en materia cooperativa, que permitan preservar la existencia de las cooperativas y el control por parte de sus socios y que faciliten el acceso a las mujeres (por ejemplo, que reduzcan el costo de registrar la propiedad de la tierra); ii) mejorar la prestación de servicios a las cooperativas en el plano local mediante el empleo de expertos en cooperativas; iii) ayudar a las cooperativas a afrontar los cambios y la competencia impuestos por la economía de mercado, el proceso de globalización, y los cambios tecnológicos; iv) reconocer la legitimidad del modelo de empresa cooperativa (por ejemplo, incluir el modelo cooperativo en los planes de estudio y los programas de formación empresarial); v) asegurar inversiones en cooperativas; vi) colaborar con la recolección de datos estadísticos que se proyecten en las políticas y programas gubernamentales, el sector privado y el propio movimiento cooperativo y, por último, vii) asociarse a las cooperativas para promover el trabajo de intercambios de mejores prácticas y las lecciones extraídas (ídem:21).

\section{CONSIDERACIONES FINALES}

Los resultados encontrados a partir de la percepción de las mujeres sobre los obstáculos en la participación de las mismas mujeres en la vida cotidiana de las cooperativas y las formas de revertirlos puede resultar en acciones más efectivas para promover la participación, tanto a través de las acciones de las cooperativas como a través de acciones y políticas públicas goberanamentales, de manera que las cooperativas puedan funcionar como espacios de fomento del cambio cultural sobre la división sexual del trabajo (CUDECOOP, 2017).

En las conversaciones realizadas con las mujeres en el momento de aplicación de las encuestas se descubrió que la mayoría tiene contacto con la cooperativa a través de la relación más directa del esposo. Sin embargo, no hubo una pregunta específica en la encuesta que capturara esta situación, así como sus limitaciones e influencias en la participación de las mujeres. En este sentido, sería importante tener una pregunta más objetiva sobre ¿qué miembros (hijo/a; esposa; esposo) de la familia están asociados con la cooperativa? ¿Cómo se siente [la mujer] acerca de los asociados? ¿Estar efectivamente asociado [o no] interfiere en su participación [de las mujeres]? Teniendo en cuenta que muchas mujeres hoy en día son jefas de hogar, las investigaciones 
también deben incluir preguntas sobre el estado civil de las mujeres y su vínculo con las cooperativas.

Al mismo tiempo, el cuestionario aplicado no capturó la participación de las mujeres en el producto / servicio que son intercambiados entre la familia y la cooperativa; por ejemplo, el tiempo que dedica a la producción de frutas, verduras y hortalizas y a los productos que entregan a la cooperativa. A menudo, consciente o inconscientemente, autoritarias o participativas, las familias organizan un trabajo productivo y trabajan con la cooperativa, y determina el miembro de la familia que está registrado en la cooperativa, donde en la mayoría de las situaciones segué siendo el hombre, mismo que las mujeres trabajen conjuntamente, o mismo más que los hombres en la producción y procesamiento en los productos comercializados en la cooperativa. Dada la realidad sexista de la sociedad, la cooperativa acaba siendo un espacio más masculino, por lo que en su mayoría son los asociados son hombres. A partir de los datos no es posible identificar cómo se organiza la pareja en relación con la cooperativa, quién forma parte de la cooperativa, quién participa en las negociaciones directas con la cooperativa, lo que ciertamente tiene implicaciones para la participación de las mujeres en la cooperativa. Estas reflexiones surgen porque cuando se les preguntó acerca de la relación con la cooperativa, muchas mujeres comentaron estar en pareja y que el esposo era el cooperado, afirmando que no sabrían cómo responder el cuestionario.

Se entiende que las mujeres participan en la cooperativa, pero que la percepción de esta participación ocurre, o se refuerza, en el momento en que participan más activamente en la producción del producto o servicio que se negocia con la cooperativa, ya sea financiamiento, crédito, productos alimenticios o al participar en las actividades de la cooperativa, ya sea en consejos, comité directivo, eventos, etc. Los resultados de la encuesta también señalan la necesidad de capacitación y acceso a la capacitación, basada en cursos de gestión para mujeres, situaciones también presentes en otras investigaciones mencionadas en este artículo.

La investigación del Cudecoop (2017) señala que los espacios de dirección son terrenos gobernados por varones y que se les hace difícil atravesar la barrera ya que ellos no quieren asumir tareas naturalizadas para mujeres, siendo necesario un cambio cultural en las cooperativas. En este sentido, una posibilidad que se presenta es garantizar una ley de cupo de género en las cooperativas, para colaborar en un intento de superación de la inequidad dentro de los espacios de decisión; "pero el cambio es más profundo y a nivel de la cultura general” (ídem: 11).

Los datos de nuestra investigación muestran que hay poca participación de mujeres jóvenes en los comités y consejos directivos, lo que representar ser 
un problema para reemplazar al personal femenino de las cooperativas, con vistas a ocupar funciones a largo plazo. Las mujeres mencionaron propuestas que pueden contribuir a esta tarea e involucrar a segmentos más jóvenes, lo que implica capacitación y motivación, creación de instrumentos legales en las reglas operativas de la cooperativa que contribuyan a la participación de mujeres, guarderías y condiciones que faciliten la movilidad para actividades cooperativas, entre otras sugerencias.

La presente investigación acabó generando datos importantes en el debate sobre las limitaciones encontradas en la participación de las mujeres en las cooperativas, que pueden generar nuevas demandas; por ejemplo, la posibilidad de pensar la realidad de la región del Mercosur, dentro del alcance del grupo de género del REAF Mercosur. Es, sin duda, una oportunidad para pensar la participación de las mujeres en las cooperativas y estimular la generación de acciones públicas y políticas públicas más precisas en la promoción del empoderamiento femenino en las actividades directivas de las cooperativas.

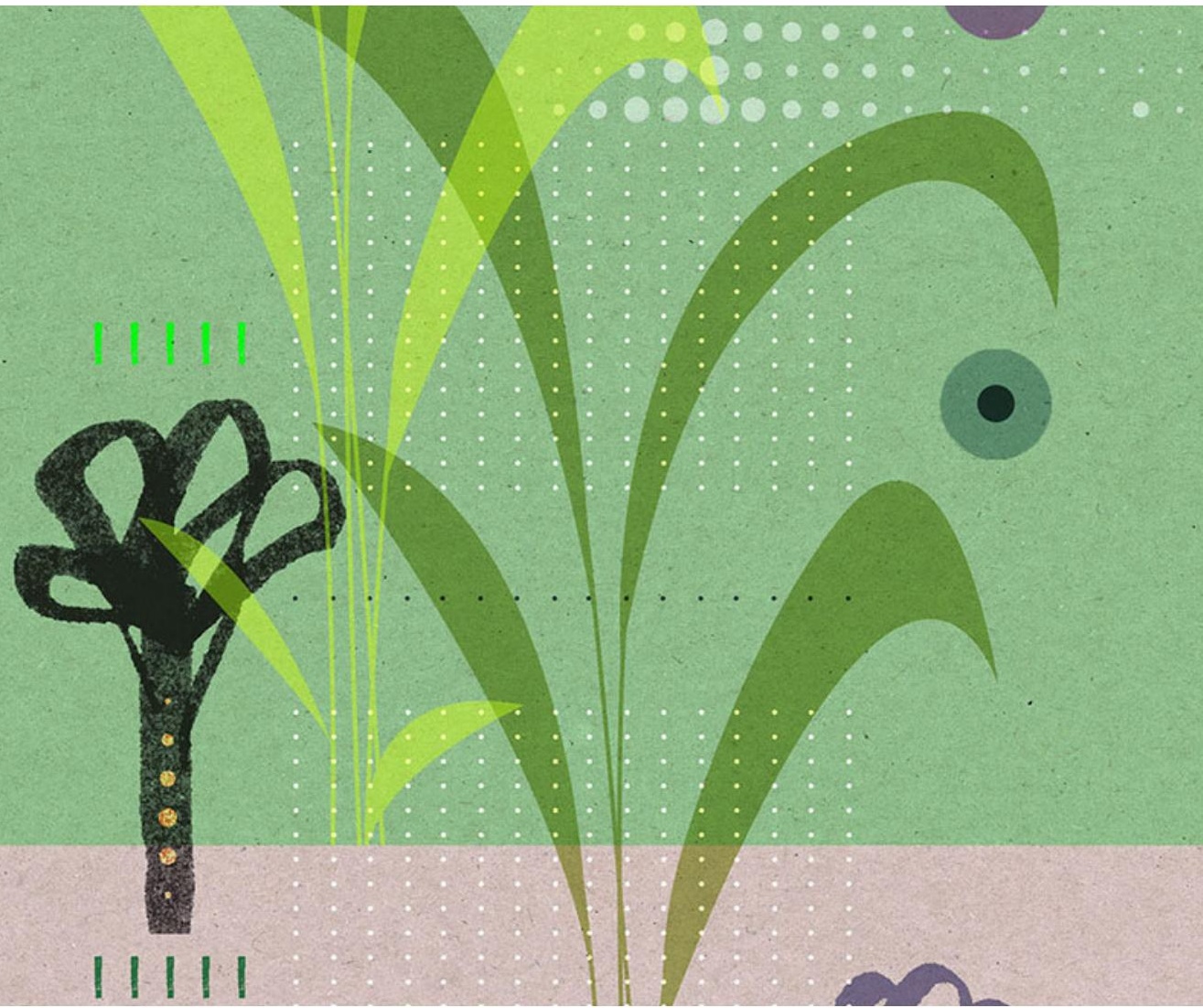




\begin{abstract}
Notas
' La investigación contó con apoyo de la Chamada Universal MCTI / CNPq n 01/2016; Chamada PRPPG / UNILA no 110/2018 y Chamada PRPPG / UNILA no 137/2018.

${ }^{2}$ Un agradecimiento especial a los estudiantes de la Unila que fueran tan dedicados a la recolección de datos, sin los cuales los siguientes análisis no habrían sido posibles. También se destaca el apoyo de Unicafes y Reaf Mercosur en el diálogo sobre las adaptaciones realizadas al cuestionario Cudecoop, en especial Geise Assis Mascarenhas, Lautaro Viscay y Maíra Figueira, quienes desde el intercambio de ideas contribuyeron fuertemente a la investigación.

${ }^{3}$ Un informe con todos los dados de la investigación se encuentra disponible en el sitio de la Organización de las Naciones Unidas para Alimentación e Agricultura (FAO): https://bit.ly/3eZWSzM. Acceso en: 22 feb. 2020.

${ }^{4}$ El Sistema Cooperativo Cresol nació en Francisco Beltrão, en el suroeste de Paraná, Brasil, donde también está instalada la sede de Central Cresol, hace más de dos décadas. Hoy se destaca como una de las principales cooperativas de crédito en Brasil, con más de 200 mil familias cooperativas en diez estados brasileños con expansión a nuevas áreas (Paraná, Santa Catarina, Rio Grande do Sul, Minas Gerais, Espírito Santo, Goiás, Rondônia, São Paulo, Río de Janeiro y Amazonas). Informaciones disponibles en: https://bit. ly/3eZBcDT. Acceso en: 22 feb. 2020
\end{abstract}

\section{Referências Bibliográficas}

BRUNO, R.; WESZ JÚNIOR, V.; BORDALO, C. A; AQUINO, S. L.; JALIL, L. Razões da participação das mulheres rurais em grupos produtivos. IN: NEVES, D. P.; MEDEIROS, L. S.; (orgs). Mulheres camponesas: trabalho produtivo e engajamentos políticos. Niterói: Alternativa, 2013. p. 217-236

BUENO, V. K.; KNUPPEL, M. A. C. A participação da mulher no cooperativismo: um estudo a partir de pesquisas em relação à temática. Publicado em 2016. Disponible en: https://bit.ly/2Wc3xye. Acceso en: 20 feb. 2020.

CARRETERO, M. J.; AVELLO, G. La participación de las mujeres en las cooperativas agrarias Estudio de diagnóstico y análisis acerca de las barreras para la participación de las mujeres en los órganos de gestión de las cooperativas del sector agroalimentario. Espanha: Fundación Mujeres, 2011. Disponible en: https://bit.ly/2xYUeKf. CONFEDERACIÓN URUGUAYA DE ENTIDADES COOPERATIVAS (CUDECOOP). Análisis e interpretación de la encuesta realizada en el Encuentro Nacional de Mujeres Cooperativistas, 21 octubre de 2017.
DUGUID, F.; WEBER, N. Gender equality and women's empowerment in co-operatives - a literature review. Bélgica: International Co-operative Alliance (ICA), 2016.

GRADE, M. S.; BASSO, D. O cooperativismo enquanto instituição para o enfrentamento à desigualdade de gênero no meio rural. Revista Orbis Latina, v.9, n. 1, Foz do Iguaçu/ PR (Brasil), Janeiro - Junho de 2019. p. 157-171. Disponível no website https://bit. ly/2SiltpY. Acceso en: 20 fev. 2020.

JALIL, L. M. As Flores e os Frutos da luta: O significado da organização e da participação política para as Mulheres Trabalhadoras Rurais. Tese de Doutorado em Ciências Sociais. Instituto de Ciências Humanas e Sociais, CPDA. Seropédica: UFRRJ, 2013.

$\mathrm{MAYO}, \mathrm{Ed}$. Breve historia de la cooperación y la mutualidad. Co-operatives UK, Manchester, 2017.

\section{ORGANIZACIÓN INTERNACIONAL} DEL TRABAJO (OIT). Avanzar hacia la igualdad: el camino cooperativo Oficina Internacional del Trabajo - Ginebra: OIT, 2016. 
REUNION ESPECIALIZADA SOBRE AGRICULTURA FAMILIAR (REAF)

Mercosur: una década de coproducción de políticas públicas entre el estado y la sociedad civil. 2016. Disponible en formato electrónico pdf: https://bit.ly/3cTCkHx.

SAGGIN, A.; ALVES, A. F. Participação das mulheres cooperativistas do Paraná em movimentos sociais, político e econômicos. Revista Orbis Latina, vol.9, $\mathrm{n}^{\circ}$ 1, Foz do Iguaçu/ PR (Brasil), Janeiro - Junho de 2019. p.27 -42. Disponible en: https://bit. ly/3eTuvDo.

SCHINCARIOL MCMURTRY, L. \& MCMURTRY, J., 2015. Advancing gender equality: The co-operative way, Geneva, Switzerland: International Labour Organization. Disponible en: https://bit. ly/2W6aqRH.

SCOTT, J. Gênero: uma categoria útil de análise histórica. Revista Educação e Realidade, Porto Alegre, v. 20, n. 2, p.71-99, jul./dez. 1995. Disponible en: https://bit. ly/3aP8Ngy.

SOUZA, J. O.; DE MARCH, Kety Carla. Belas, recatadas e do lar?: empoderamento das mulheres no cooperativismo. Publicado en 2018. https://bit.ly/2yVejRO.
TRIVIÑOS, A. N. S. Introdução à pesquisa em ciências sociais: a pesquisa qualitativa em educação. São Paulo: Atlas, 175 p. 1987.

UNIÓN DE COOPERATIVAS DE AGRICULTURAY ECONOMİA FAMILIAR Y SOLIDARIDAD (UNICAFES). Histórico. Disponible en: https://bit.ly/2SxqD1B.

VEDANA, R. Empoderamento feminino na agricultura: um estudo de caso na Lar Cooperativa Agroindustrial (Paraná). 2020, 89 p. Dissertação de Mestrado (Mestrado em Economia) - Universidade Estadual do Oeste do Paraná, 2020.

VOLLES, A.; BONA, A. N. Cooperativas de crédito rural: a inserção das mulheres nos conselhos. Publicado em 2016. Disponível em: https://bit.ly/35cI3Wl.

ZIMMERMANN, S. A.; GARCIAS, M.; BASSO, D. Participação das mulheres em espaços cooperativos. $57^{\circ}$ Congresso da Sociedade Brasileira de Economia, Administração e Sociologia Rural, 21 a 25 de julho de 2019. Anais Eletrônicos - ISBN: 978-85-98571-18-8. Disponible en: https:// bit.ly/3fowRAr.

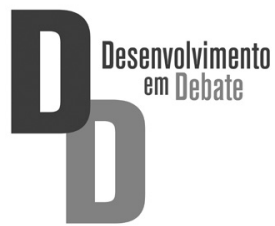

